\title{
Improving the Latency Value by Virtualizing Distributed Data Center and Automation in Cloud.
}

\author{
C.Eng. (Mrs.).Nusrath Sultana B.Tech, AMIEI. \\ Assistant professor, Global Institute of Engineering and Technology, Affiliated to JNTU-H, India
}

\begin{abstract}
Organization today are leveraging the benefits of cloud computing to increase flexibility, agility and reduce cost however that flexibility can also pose networking challenges by moving application offsite, companies need good network connectivity between a data center site and a cloud provider so that user don't experience performance degradation. Good connectivity comes in two forms necessary bandwidth and low latency. Distributed datacenter improves services access latency and bandwidth. Virtualized cloud data center enables IT organization to share compute resources across multiple applications and user group in a much more dynamic way than is possible in traditional environment where application, middleware and infrastructure are tightly coupled and resource allocation are highly static. The goal is to enable users to reduce the cost and complexity of application provisioning and operations in virtualized data centers. Cloud environments at the same by automation liberate the operational management from the burden of manual process.
\end{abstract}

\section{Introduction}

Decades of software and hardware purchases made across the enterprise have left systems residing on grossly underused infrastructure. In fact, companies on average use only $15 \%$ to $20 \%$ of available server and storage capacity. Supporting this highly complex, fragmented, and inefficient environment can cost up to ninetenths of your annual IT budget. Updates and fixes get done manually, leading to errors, security problems, and infrastructure downtime. Even worse, time to market and customer satisfaction can suffer. In this paper we will see how best we can reduce the latency and improve the bandwidth by virtualizing the distributed data center and automatic configuration.

\section{Centralized data center Vs Distributed data center}

The debate over centralized versus distributed data center , is seem to the pendulum swinging back and forth, some companies still consider to keep centralized data centers. This paper presents distributed data center over centralized data center. Distributed data center will have much lower latency characteristics per application and per services than those that are deployed centrally. Service provider will gain financial benefit by distributing their data centers, positioning select services close to the customers who will use them. There are a number of different storage model in use today such as (1) Storage over IP (SoIP). (2) Fiber Channel over Ethernet (FCoE). (3) Traditional Fiber Channel. All require a network that offers low latency and high availability. Deploying distributed datacenter benefit is Wide Area Bandwidth Saving. When application data is duplicated at multi data centers, clients go to the available data center in the event of catastrophic failure at one site. Data center can also be used concurrently to improve performance and scalability. Once the content is distributed to multiple data centers. We need to manage the request for the distributed content, manage the load by routing user request for content to the appropriate data center, selecting the appropriate data center (which is based on server availability, content availability), network distance from client to the PC's and other parameters.[8]

\subsection{Benefits of Distributed Data centers:}

1. Archiving data for protection against data loss and corruption, or to meet regulatory requirements

2. Performing remote replication of data for distribution of content, application testing, disaster protection, and data centre migration

3. Providing non-intrusive replication technologies that do not impact production systems and still meet shrinking backup window requirements

4. Protecting critical e-business applications that require a robust disaster recovery infrastructure. Providing real-time disaster recovery solutions, such as synchronous mirroring, allow companies to safeguard their data operations by: 


\subsection{The relationship between bandwidth consumed per subscriber to the cost of delivering it:}

The cost per application increases linearly for services hosted in centralized data center while it remains relatively stable for applications hosted in a distributed data center.

Cost per application, varying number of subscribers/application

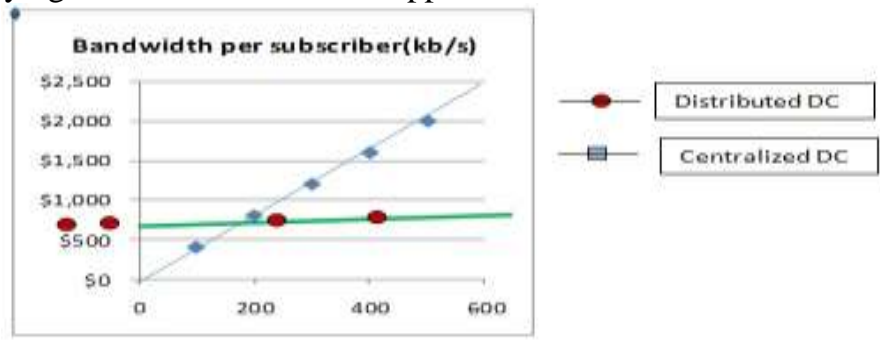

Figure: 1

2.3 The relationship between the cost of delivering an application and the growth rate in the number of subscribers using it:

Here the cost advantage of the distributed data center is significant as the application becomes more popular. Network is a critical resource in the cloud to automate and more effectively manage and distribute resources for better performance.

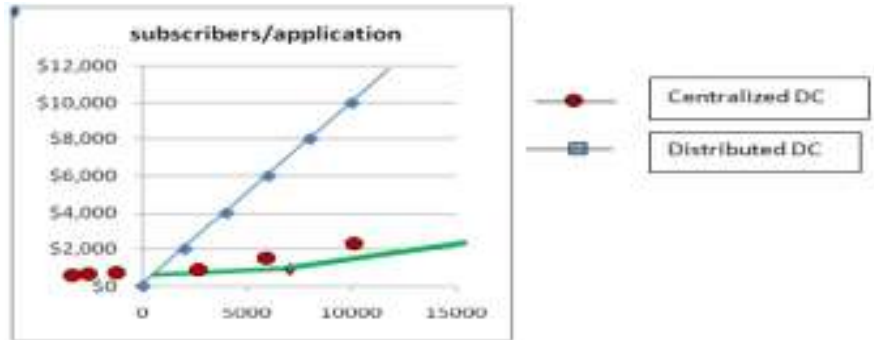

Figure: 2

\section{Virtualized cloud datacenters}

Virtualization:_A transparent abstraction of computer resources making a physical resource appears as multiple logical ones. Virtualization increases utilization of server, reduce data center footprints, and minimize power requirement[6].For example VmWare offers virtual private networking capabilities as part of its VShield Suite of products. Vshield protects application in the virtual data center against network based threats[2]

\subsection{Benefits of Virtualization:}

1. Enhance service level: provision services and resources to the business more quickly; often they are able to reduce the cycle time from service request to service availability from multiple weeks to just a few days or hours in the case of self service solution.

2. Cost Saving: Consumption based metering and capacity planning helps IT spending with business needs and ensures optimal use of available system application and staff resource. Virtualization greatly reduces capex and opex, the ratio of administrators to physical and virtual server from 1:30 to 1:1000+ results in significant to IT product and cost saving.

3. Improve operational efficiency: Significant expansion of automation and orchestration strategies across the internal data center environment improves IT operational efficiency.

4. Increase availability and reduce energy consumption: power consumption will always be less after virtualizing as a result of computing consolidation and physical reduction of the amount of IT equipment. Which is a contribution to green computing?

5. Improve overall business flexibility and agility: By providing better optimize end-to-end application performance and availability by reducing downtime, maintaining more consisting patching and security processes and improving the ability to diagnose and reduce the root cause of problem overall business flexibility will improve.

\section{Network of cloud to improve latency:}

Here we are architechuring the multi cloud support and are so found on zero modification of server and applications where we have a freedom to use a cloud that is "closer" without changing the configuration where we can achieve our task with low latency, high SLA and better pricing. By virtualizing the distributed data center we can build on demand virtual data center in multitenant environment which rapidly provision 
application to meet business needs. Now we need a network in the network pool. (Network pool is a collection of virtual machine) network where traffic at each network is isolated at layer 2 when it is available to be consumed by organization to create organization network and Vapp network. Here we are making data centre behave like a cloud(share pool of services), at layer 2 instead of having a physical partition we go by logical partition which enables secure sharing of data between data centers and extend it to WAN to connect with other cloud.

Jupiner simplifies the data center network and eliminates layer of cost and complexity with a 3-2-1 data center network architecture using technologies such as virtual private LAN services(VPLS).Network Virtualization on juniper network MX Series 3D universal edge router, Virtual chassis on juniper network EX series Ethernet switches, and Juniper network QFabric architecture on QF X series product family. See for example The Cloud Ready Data Center Network of Jupiner Network[3].

By using VPLS, MPLS, VPRN, PBB we can address the challenges of multi tenancy, mobility, scalability, High availability, low latency [1].

\subsection{Components of a virtual network:}

1. Network hardware such as switches and network adapter also know as network interface cards (NIC).

2. Network element such as firewalls and load balancers

3. Network such as virtual LAN (VLAN) and containers such as VM and Solaris container.

4. Network storage devices.

5. Network M2M (machine to machine) elements such as telecommunication 4G HLR and SLR devices.

6. Network mobile element such as laptops, tablets and cell phone.

7. Network media such as Ethernet and fiber channel.

\subsection{The importance of network bandwidth for storage:}

Cloud Computing offers IT far greater flexibility in how it delivers services but that flexibility can pose networking and storage challenges [5].Storage networks with plenty of bandwidth are also a valuable asset in virtual infrastructures. The required amount of storage bandwidth depends not only on the number of transactions but also on the transaction size. Windows File Servers, for example, tend to use tiny transactions to access the storage, and database servers use medium-size transactions.

In both cases, these workloads will likely be limited by the storage's transaction rate, long before network bandwidth becomes a factor. For low-utilization, easy-to-virtualized VMs, the storage network won't be limiting, even at GbE speeds. For the more critical, resource-intensive VMs, however, you'd better make sure you have dozens of spindles or a fair amount of solid-state drives before you start demanding faster storage networks

\subsection{Backups for network bandwidth:}

As we've moved from a 9-to-5 working day to the always-on Internet age, the working day overlaps with the backup window -- which is when organizations back up their servers, usually during off peak times. Now companies need full-speed performance during a backup, so the network better not be saturated.[6]

Backups involve large transactions and can quickly fill a network. As such, it's common sense to have a nice, big pipe to carry the data. If that's the case, the storage disks will be the limiting factor, rather than the link itself. More specifically, if backup tool uses agents inside the VMs, then we require big pipes into the virtual hosts to avoid a blowout during the backup windows. (Also make sure your hosts have ample CPU and RAM to cope with this load spike.). Therefore, connecting the backup server to the storage array, using the biggest pipe available, is definitely a good idea. If we have a fast network between backup server and main storage, it make sense to have a slower network for hosts? If new equipments are buyed, then probably not. The incremental cost of fast network ports won't be much, and over time, the demand for bandwidth will likely increase. To solve the problem of backup speed, we probably buy faster ports for server and storage.

IT pros often cite virtualization and backups as reasons why they need more network bandwidth, but we don't necessarily need a $10 \mathrm{GbE}$ network to maintain a high level of performance .On the surface, it makes sense that a virtual infrastructure needs plenty of network bandwidth. Let's say that an organization just consolidated 20 physical servers, each with two Gigabit Ethernet (GbE) ports, into one virtual host. Surely that means the host needs more than a few GbE ports?

More on network bandwidth in virtual data centers $10 \mathrm{GbE}$ : Cutting cabling, boosting virtualization network management, Virtual networking design, configuration and management guide.More on network bandwidth in virtual data centers $10 \mathrm{GbE}$ : Cutting cabling, boosting virtualization network management, Virtual networking design, configuration and management guide.The reality is that almost all of those physical hosts didn't use their full network bandwidth, apart from tiny bursts. So sharing a GbE port among a dozen virtual machines (VMs) won't be a problem. Virtualization tends to increase the average utilization of these ports from less than $1 \%$ to $5 \%$ or $10 \%$. The VMs just don't need a lot of network bandwidth. 
That said the virtualization hosts require fast ports, mostly for transferring VMs between hosts. Moving $16 \mathrm{~GB}$ worth of a VM's contents during a powered-on live migration will saturate a GbE port for a few minutes. The issue is exacerbated when migrations involve a huge amount of RAM.If virtual host with 128 GB of RAM is filled to capacity, it may take a half an hour or more to migrate all of the VMs using a single GbE port. If we migrating these VMs because of an impending physical failure, it will feel a lot longer. (Just imagine that feeling when a host with terabytes of memory is about to fail.) But emptying the same 128 GB host with $10 \mathrm{GbE}$ connection will take about five minutes, reducing the risk of a VM outage because of a virtual host failure.

\section{Automation and orchestration of cloud}

Automation and orchestration are often lumped under the same heading and no wonder their roles are often confused. For some the two words are synonymous: for other the phase"automation and orchestration" is treated as a single word. [4]. Automation is generally associated with a single task where as orchestration is associated with a workflow process for several tasks. Save time and money on infrastructure management processes such as asset tracking, application and patch provisioning, code deployment and rollback, monitoring and failover, and assigning computing resources. The virtualization can reduce the provisioning time but not installation time. The IT staff uses labor - intensive management tools and manual script to control and manage a data center infrastructure. But they won't be able to keep pace with continuous stream of on figuration changes associated with clouds dynamic provision and virtual movement nor can they maintain access and security changes. That is why process automation becomes so important in a cloud. A shift to standardized service centric delivery model, pared with extensive use of automation and orchestration technologies, can significantly improve IT operation productivity and end-to-end service levels [7].

Automation and orchestration helps to make infrastructure changes more rapidly, but these changes have to be recorded nearly simultaneously so that orchestration function has up-to-date configuration data needed to make decision, such as CPU allocation and storage. The rapidity of change stemming from automation and self-service in cloud environments requires a more efficient approach to configuration management and change management inside the IT organization Configuration Management Database (CMDB) can record these changes in real time.

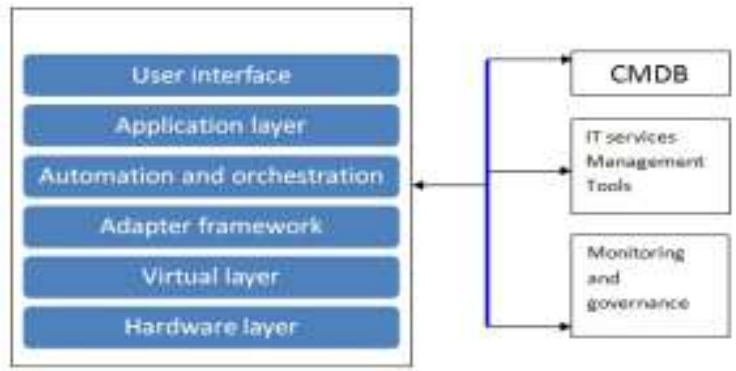

Figure: 3

\section{Conclusions And Future Work}

In cloud computing now a days the more concerned area is latency value .In this paper we try to solve the latency value by making use of virtualized distributed data centres and at the same time by automating the configuration which will avoid the manual error and complexity to manage servers and virtual network by server manager and network manager. Effective automation virtual system attributes to support for heterogeneous physical and virtual environment; simplify, integrate and standardize workflow; ability to integrate infrastructure, operating system, and application software; self-serve provisioning interface. Without automation and orchestration tools, it has to manually reprovision and optimize resources to reflect even the smallest changes in an environment. The future work emphasis on enabling effective energy management through automation and real time monitoring.

\section{References}

[1] Creating cloud ready data center-Technology white paper page 1-7.

[2] Blog.vmware.com how to install and configure vshield manager for use with vmware vcloud director.

[3] Jupiner Network-Cloud Ready Data Center Network.WWW.jupiner.net.

[4] Bill Claybrook, E-Zine volume 1,no.3, Tools to unlock a private cloud potential page 14-18.

[5] Bob Plankers, E-zine volume 1,No.3 IT Without Borders, page 8-10.

[6] Alastair Cooke,serarchvirtualization.techtarget.com,hpw much network bandwidth is enough for virtualized data centers.

[7] Tim Grieser and Mary Johnston Turner-Automated provisioning and orchestration is critical to effective private cloud operation.

[8] www.cisco.com .Design Zone for Data Centers. 